\title{
KIF14 promotes cell proliferation via activation of Akt and is directly targeted by miR-200c in colorectal cancer
}

\author{
ZAO-ZAO WANG ${ }^{*}$, JIE YANG $^{*}$, BEI-HAI JIANG, JIA-BO DI, PIN GAO, LIN PENG and XIANG-QIAN SU \\ Key Laboratory of Carcinogenesis and Translational Research (Ministry of Education), \\ Department of Gastrointestinal Surgery IV, Peking University Cancer Hospital and Institute, Beijing 100142, P.R. China
}

Received April 24, 2018; Accepted August 9, 2018

DOI: $10.3892 /$ ijo.2018.4546

\begin{abstract}
As a mitotic kinesin, kinesin family member 14 (KIF14) has been reported to serve oncogenic roles in a variety of malignancies; however, its functional role and regulatory mechanisms in colorectal cancer (CRC) remain unclear. In the present study, KIF14 was observed to be markedly overexpressed in CRC, and this upregulation was associated with tumor size and marker of proliferation Ki-67 immunostaining scores. Gain- and loss-of-function experiments were applied to identify the function of KIF14 in CRC progression. In vitro and in vivo assays revealed that KIF14 promoted CRC cell proliferation and accelerated the cell cycle via activation of protein kinase B. In addition, the present study investigated the potential mechanisms underlying KIF14 overexpression in CRC. Bioinformatics analyses and validation experiments, including reverse transcription-quantitative polymerase chain reaction, western blotting and a Dual-Luciferase reporter assay, demonstrated that, in addition to genomic amplification and transcriptional activation, KIF14 was regulated by microRNA (miR)-200c at the post-transcriptional level. Rescue experiments further demonstrated that decreased miR-200c expression could facilitate KIF14 to exert its pro-proliferative role. The expression of miR-200c was negatively correlated with KIF14 in CRC specimens. Collectively, the findings of the present study demonstrated the oncogenic role of KIF14 in colorectal tumorigenesis, and also revealed a complexity of regulatory mechanisms mediating KIF14 overexpression, which may provide insight for developing novel treatments for patients with CRC.
\end{abstract}

Correspondence to: Professor Xiang-Qian Su, Key Laboratory of Carcinogenesis and Translational Research (Ministry of Education), Department of Gastrointestinal Surgery IV, Peking University Cancer Hospital and Institute, 52 Fucheng Road, Haidian, Beijing 100142, P.R. China

E-mail: suxiangqian@bjmu.edu.cn

*Contributed equally

Key words: kinesin family member 14, colorectal cancer, cell proliferation, cell cycle, microRNA-200c

\section{Introduction}

As one of the most common types of gastrointestinal malignancies, colorectal cancer (CRC) remains a major public health problem worldwide $(1,2)$. In China, CRC ranks as the fifth leading cause of cancer-associated mortality nationwide, and its incidence is increasing annually (3). At present, surgical resection is still the most effective treatment for patients with CRC (4). The clinical outcomes of CRC have gradually improved due to numerous developments in screening, detection and adjuvant therapy; however, the prognosis of patients with advanced CRC remains poor due to tumor metastasis and recurrence $(5,6)$. Recent studies have revealed that certain genetic and epigenetic abnormalities are strongly associated with the clinical outcomes of CRC $(7,8)$. Therefore, identifying the molecular mechanisms involved in the onset and progression of CRC is required to improve the therapeutic effectiveness of treatments for CRC.

The kinesin superfamily proteins (KIFs) are essential microtubule-dependent molecular motors that transport a variety of cargo, including organelles, transcripts and proteins in an ATP-dependent manner $(9,10)$. At present, $>45 \mathrm{KIFs}$ have been identified in mammals and are classified into 14 subfamilies (11). According to the position of the motor domain, KIF members are divided into $\mathrm{N}$-kinesin, $\mathrm{C}$-kinesin and $\mathrm{M}$-kinesin sub-groups (11). Kinesin family member 14 (KIF14), located on chromosome 1q32.1, is an N-type kinesin and belongs to the kinesin-3 superfamily $(12,13)$. KIF14 has been reported to contain four conserved functional domains, including an $\mathrm{N}$-terminal extension domain that binds with protein regulator of cytokinesis 1 (14), a motor domain required for movement along the microtubule via the hydrolysis of ATP (15), a forkhead-associated domain which regulates phosphorylation $(15,16)$, and a stalk and tail domain for the interaction with citron kinase $(16,17)$. The functions of KIF14 have been associated with a variety of biological processes $(18,19)$. Previous studies have demonstrated that the depletion of KIF14 may induce the incompletion of cytokinesis and lead to multinucleation $(19,20)$. Additionally, the homozygous mutation of KIF14 may result in autosomal recessive primary microcephaly $(21,22)$.

Aberrant overexpression of KIF14 has been reported in numerous types of cancer (23-31); the oncogenic effects of KIF14 are also diverse. Overexpressed KIF14 promotes cell 
Table I. Association between the expression of KIF14 and clinicopathological features in 36 patients with CRC.

\begin{tabular}{|c|c|c|c|}
\hline Characteristics & No. of patients $(\%)$ & $\mathrm{KIF} 14^{\mathrm{a}}$ & P-value \\
\hline Sex & & & 0.828 \\
\hline Male & $27(75.0)$ & $1.048(0.553-4.368)$ & \\
\hline Female & $9(25.0)$ & $1.341(0.533-4.177)$ & \\
\hline Age (years) & & & 0.249 \\
\hline$<60$ & $18(50.0)$ & $0.791(0.431-5.137)$ & \\
\hline$\geq 60$ & $18(50.0)$ & $1.435(0.818-4.118)$ & \\
\hline Tumor size (cm) & & & $0.008^{\mathrm{d}}$ \\
\hline$<4$ & $12(33.3)$ & $0.696(0.338-1.240)$ & \\
\hline$\geq 4$ & $24(66.7)$ & $1.746(0.786-7.164)$ & \\
\hline Invasion & & & 0.726 \\
\hline $\mathrm{T} 2+\mathrm{T} 3$ & $12(33.3)$ & $1.197(0.639-5.330)$ & \\
\hline $\mathrm{T} 4$ & $24(66.7)$ & $1.194(0.456-3.480)$ & \\
\hline Lymphatic metastasis & & & 0.949 \\
\hline Negative & $14(38.9)$ & $1.051(0.442-8.798)$ & \\
\hline Positive & $22(61.1)$ & $1.320(0.573-3.797)$ & \\
\hline Distal metastasis & & & 0.807 \\
\hline Negative & $18(50.0)$ & $1.197(0.537-6.606)$ & \\
\hline Positive & $18(50.0)$ & $1.116(0.545-3.417)$ & \\
\hline Stage (AJCC) & & & 0.949 \\
\hline Stage II & $14(38.9)$ & $1.051(0.442-8.798)$ & \\
\hline Stage III/IV & $22(61.1)$ & $1.320(0.573-3.797)$ & \\
\hline MKI67 immunostaining score & & & $0.016^{\mathrm{c}}$ \\
\hline$<50 \%$ & $17(47.2)$ & $0.611(0.409-1.079)$ & \\
\hline$\geq 50 \%$ & $19(52.8)$ & $1.743(0.780-4.368)$ & \\
\hline
\end{tabular}

${ }^{\mathrm{a}}$ Median of relative expression, with 25 th-75th percentile in parenthesis. ${ }^{\mathrm{b}} \mathrm{P}$-values were calculated by using the two-tailed Student's t-test. ${ }^{\mathrm{C}} \mathrm{P}<0.05,{ }^{\mathrm{d}} \mathrm{P}<0.01$. KIF14, kinesin family member 14; AJCC, American Joint Committee on Cancer, 7th Edition; MKI67, marker of proliferation Ki-67.

proliferation in malignancies, including glioblastoma, lung and ovarian cancer $(23,24,30,31)$, affects cell cycle progression and cytokinesis in hepatocellular carcinoma (25) and interferes with chemosensitivity in triple-negative breast cancer $(27,29)$. The genomic gain of 1q32 has been detected in several cancer types, including CRC (32); however, the role of KIF14 in colorectal carcinogenesis remains poorly understood. Aside from its functional role, the regulation of KIF14 has not been investigated in CRC.

In the present study, it was demonstrated that KIF14 was upregulated in CRC tissues and promoted tumor cell proliferation. Abnormally overexpressed KIF14 was proposed to partly contribute to genomic amplification and transcriptional activation. Additionally, the present study revealed that microRNA (miRNA/miR)-200c, a well-reported tumor suppressor $(33,34)$, regulated the expression of KIF14 at the post-transcriptional level. To the best of our knowledge, the present study is the first to comprehensively demonstrate the expression profile and functional roles of KIF14 in CRC, and reveal that downregulated miR-200c expression may contribute to increased KIF14 expression.

\section{Materials and methods}

Ethics statement. All methods conducted in the present study and all the experimental protocols were approved by the Research Ethics Committee of Peking University Cancer Hospital and Institute (Beijing, China).

Patients and specimens. A total of 36 pairs of CRC specimens accompanied with adjacent non-cancerous tissues were obtained from patients who underwent colorectal resection at Peking University Cancer Hospital and Institute from February 2004 to November 2007. All patients enrolled in the present study were pathologically confirmed as primary colorectal adenocarcinoma by endoscopic biopsy, and had not received adjuvant chemotherapy, radiotherapy, or immunotherapy prior to surgery. Tissue samples were frozen in liquid nitrogen. The MKI67 immunostaining scores of the specimens were obtained from the pathological reports and the clinicopathological features of patients are presented in Table I. Prior to surgery, written informed consent was obtained from each patient. 
Table II. Primers and sequences.

A, Primers for amplification for expression vectors

\begin{tabular}{ll}
\hline Name & \multicolumn{1}{c}{ Sequence } \\
\hline pCDNA3.1-KIF14 CDS clone & $\begin{array}{l}\text { Forward: 5'-CGCGGATCCATGTCATTACACAGTACTCA-3' } \\
\text { Reverse: 5'-CCGCTCGAGTCACACCCACTGAATCCTACT-3' }\end{array}$ \\
pGL3-WT-KIF14 3'UTR luciferase reporter & Forward: 5'-CGTCTAGAACCTGATCCTTTCATTTGCCCT-3' \\
pGL3-MUT1-KIF14 3'UTR luciferase reporter & Reverse: 5'-CGTCTAGAGTCAGTGCACATAATTCCAATAGC-3' \\
& Forward: 5'-GTGAACCTTGTGTGGTGTTTTCAAGCTCT-3' \\
pGL3-MUT2-KIF14 3'UTR luciferase reporter & Reverse: 5'-ATTTACTTACAATCTCTTCCTAACAAGGA-3' \\
& Forward: 5'-AATCTTCAGTTTGTGCTTTGTAAACTC-3' \\
& Reverse: 5'-AACTACTATACAGGTACTTTAGGAAGTTCT-3'
\end{tabular}

B, RT-qPCR primers

Name

GAPDH

KIF14
Sequence

Forward: 5'-TGCACCACCACCTGCTTAGC-3'

Reverse: 5'-GGCATGGACTGTGGTCATGAG-3'

Forward: 5'-CCGACATTACAGATGCACCA-3'

Reverse: 5'-CTTCATTCCTAAGCCTACACC-3'

C, KIF14 siRNAs

\begin{tabular}{ll}
\hline Name & \multicolumn{1}{c}{ Sequence } \\
\hline KIF14 siRNA 1 & Sense: 5'-CAGCGGUGAUAUUCUUGAUTT-3' \\
KIF14 siRNA 2 & Antisense: 5'-AUCAAGAAUAUCACCGCUGTT-3' \\
& Sense: 5'-CUCAGAGCAAGUUGGAUAUTT-3' \\
KIF14 siRNA 3 & Antisense: 5'-AUAUCCAACUUGCUCUGAGTT-3' \\
& Sense: 5'-GCCCGUUUAAUAGUCAACATT-3' \\
siRNA NC & Antisense: 5'-UGUUGACUAUUAAACGGGCTT-3' \\
& Sense: 5'-UUCUCCGAACGUGUCACGUTT-3' \\
& Antisense: 5'-ACGUGACACGUUCGGAGAATT-3'
\end{tabular}

D, KIF14 shRNAs

Name

KIF14 shRNA T459

KIF14 shRNA T460

shRNA NC
Sequence

\author{
5'-CTCAGAGCAAGTTGGATAT-3' \\ 5'-CAGCGGTGATATTCTTGAT-3' \\ 5'-TTCTCCGAACGTGTCACGT-3'
}

KIF14, kinesin family member 14; CDS, coding sequence; WT, wild-type; MUT, mutation; UTR, untranslated region; RT-qPCR, real-time quantitative polymerase chain reaction; GAPDH, glyceraldehyde-3-phosphate dehydrogenase; siRNA, small interfering RNA; shRNA, short hairpin RNAs; NC, negative control.

Cell culture and transfection. The human CRC cell lines (SW620, SW480, HCT116, RKO and LoVo) and 293T cells were purchased from the American Type Culture Collection (ATCC; Manassas, VA, USA). Cells were maintained in either Dulbecco's modified Eagle's medium or RPMI-1640 medium
(HyClone Laboratories; GE Healthcare Life Sciences, Logan, UT, USA) supplemented with $10 \%$ fetal bovine serum (FBS) in a humidified atmosphere with $5 \% \mathrm{CO}_{2}$ at $37^{\circ} \mathrm{C}$.

To overexpress KIF14, the KIF14 coding sequence was amplified from cDNA template of RKO cells and cloned into 
the pcDNA3.1 vector (P3.1-KIF14 CDS for short), which was verified by DNA sequencing. The miR-200c-binding sequence of the KIF14 3'-untranslated region (UTR) was inserted into the pGL3-Control construct (Promega Corp., Madison, WI, USA) and was denoted as pGL3-KIF14 wild-type (WT) 3'UTR. A Takara MutanBEST kit (Takara Bio, Inc., Otsu, Japan) was applied to generate mutant (MUT) constructs with a single (MUT1/MUT2) or double (DOU MUT) miR-200c core binding site deletion; mutants were denoted as pGL3-MUT1KIF14, pGL3-MUT2-KIF14 or pGL3-Double-MUT-KIF14, respectively. The designed primers are summarized in Table II.

miR-200c mimics (assay ID MC11714) or inhibitor (assay ID MH11714), and a control oligo (mi-NC) (cat. no. 4464058) were obtained from Invitrogen (Thermo Fisher Scientific, Inc. Waltham, MA, USA). KIF14 small interfering (si)RNAs and lentiviruses expressing KIF14 short hairpin (sh)RNAs, together with their negative controls were purchased from Shanghai GenePharma Co., Ltd. (Shanghai, China).

Plasmids, siRNAs, miR-200c mimics or inhibitor, and their corresponding controls were transiently transfected into cells by using Lipofectamine ${ }^{\circledR} 3000$ (Invitrogen; Thermo Fisher Scientific, Inc.) according to the manufacturer's instructions.

MTT assay. Cells were seeded into 96-well plates at the concentration of $5 \times 10^{3}$ cells/well. At the indicated time-points, $20 \mu \mathrm{l}$ MTT was added to each well. Following incubation, dimethyl sulfoxide was used to solubilize the crystals. The absorbance was recorded at $570 \mathrm{~nm}$ using a Model 680 microplate reader (Bio-Rad Laboratories, Inc., Hercules, CA, USA).

Plate colony formation assay. At 24 or $48 \mathrm{~h}$ after transfection, cells were counted and seeded into 6-well plates at the concentration of 200 cells/well, and then maintained in complete medium, which was refreshed every 3 days. Following incubation for 2 weeks, colonies were notably visible. Subsequently, the cells were fixed with $100 \%$ cold methanol for $15 \mathrm{~min}$, stained with $1 \%$ crystal violet for $20 \mathrm{~min}$ at room temperature and washed with PBS at least three times. The colony numbers were then counted.

Cell cycle. At $48 \mathrm{~h}$ after transfection, cells were harvested and fixed with $70 \%$ cold ethanol at $-20^{\circ} \mathrm{C}$. Then, $1 \times 10^{6}$ cells were stained with $0.5 \mathrm{ml}$ propidium iodide $(\mathrm{PI}) / \mathrm{RNase}$ staining buffer (BD Pharmingen; BD Biosciences San Jose, CA, USA) for $15 \mathrm{~min}$ at room temperature, and the cell cycle distribution was examined by flow cytometry. The extent of PI staining was detected by a BD FACSCalibur Flow Cytometer (BD Biosciences). Additionally, DNA histograms of the cell cycle distribution were processed using FlowJo Analysis Software v.8.7.3 (FlowJo LLC, Ashland, OR, USA).

5-Ethynyl-2-deoxyuridine (EdU) incorporation assay. The effect of KIF14 on cell proliferation was also determined via an EdU incorporation assay using the Cell-Light ${ }^{\mathrm{TM}}$ EdU In Vitro Imaging kit (Guangzhou RiboBio Co., Ltd., Guangzhou, China). Cells $\left(1 \times 10^{4}\right.$ cells/well) were seeded into a 96-well plate and cultured for $24 \mathrm{~h}$, the indicated cells were incubated with $50 \mu \mathrm{M}$ EdU for $2 \mathrm{~h}$ at $37^{\circ} \mathrm{C}$ after $24 \mathrm{~h}$ post-transfection. Following several washes, the cells were fixed with $4 \%$ formaldehyde for $30 \mathrm{~min}$ and treated with $0.5 \%$ Triton X-100 for an additional $20 \mathrm{~min}$ at room temperature. Then, permeabilized cells were incubated with $1 \mathrm{X}$ Apollo reaction cocktail for $30 \mathrm{~min}$ at room temperature in the dark. Then, the cells were stained with Hoechst $33342(5 \mu \mathrm{g} / \mathrm{ml})$ for $30 \mathrm{~min}$ away from light. The proliferative activity of treated cells was visualized under a Zeiss confocal microscope LSM 700 (Carl Zeiss AG, Oberkochen, Germany). Three replicates were performed for each group, and five fields per well were selected for counting. The rate of EdU incorporation was calculated as the ratio of EdU-positive cells (green cells) to the total number of Hoechst 33342 positive cells (blue cells).

Western blotting. Transfected cells were harvested and lysed in radioimmunoprecipitation assay buffer with a protease inhibitor cocktail (Roche Applied Science, Penzberg, Germany). Then, the concentration of protein samples was determined with a Pierce BCA Protein assay kit (Pierce; Thermo Fisher Scientific, Inc.). Equal amounts of protein $(30 \mu \mathrm{g})$ were separated by $10 \%$ SDS-PAGE and then transferred to nitrocellulose membranes (EMD Millipore, Billerica, MA, USA). Following blocking with $5 \%$ non-fat milk for $1 \mathrm{~h}$ at room temperature, the membranes were incubated overnight at $4^{\circ} \mathrm{C}$ with primary antibodies against KIF14 (polyclonal, rabbit anti-human $\mathrm{IgG}$; 1:1,000; cat. no. ab3746; Abcam, Cambridge, MA, USA), protein kinase B (Akt; polyclonal, rabbit anti-human IgG; 1:1,000; cat. no. 9272s; Cell Signaling Technology, Inc., Danvers, MA, USA), phosphorylated-Akt (polyclonal, rabbit anti-human IgG; 1:200; cat. no. 9271s; Cell Signaling Technology, Inc.) or $\beta$-actin (monoclonal, mouse anti-human IgG; 1:3,000; cat. no. AC-15; SigmaAldrich; Merck KGaA, Darmstadt, Germany). Following washing with $1 \mathrm{X}$ PBS containing $0.05 \%$ Tween-20 for three times, the membranes were incubated at room temperature for $1 \mathrm{~h}$ with either horseradish peroxidase-conjugated goat anti-rabbit (1:2,000; cat. no. ZB-2301; OriGene Technologies, Inc., Beijing, China) or goat anti-mouse (1:2,000; cat. no. ZB-2305; OriGene Technologies, Inc.) secondary antibodies. An enhanced chemiluminescence detection kit (Amersham; GE Healthcare, Chicago, IL, USA) was applied to detect the signal. Relative protein expression levels were normalized to $\beta$-actin and quantified with Image J software (National Institutes of Health, Bethesda, MD, USA).

$R N A$ isolation and reverse transcription-quantitative polymerase chain reaction ( $R T-q P C R)$. Total RNA from freshly cultured cells or tumor samples ground in liquid nitrogen was extracted using TRIzol reagent (Invitrogen; Thermo Fisher Scientific, Inc.). Subsequently, RNA samples were quantified with a NanoDrop spectrophotometer (ND-1000, NanoDrop; Thermo Fisher Scientific, Inc.) and $1 \mu \mathrm{g}$ of total RNA was reverse transcribed into cDNA using a Reverse Transcription kit (Promega Corp.). cDNA templates were then mixed with SYBR Green PCR Master Mix (Toyobo Life Science, Osaka, Japan) and the corresponding primers; qPCR was subsequently performed on the ABI 7500 Real-time PCR System (Applied Biosystems; Thermo Fisher Scientific, Inc.). The thermocycling conditions were set to $95^{\circ} \mathrm{C}$ for $5 \mathrm{~min}$, followed by 40 cycles of $95^{\circ} \mathrm{C}$ for $10 \mathrm{sec}$ and $60^{\circ} \mathrm{C}$ for $30 \mathrm{sec}$. The subsequent dissociation stage was set to $95^{\circ} \mathrm{C}$ for $15 \mathrm{sec}, 60^{\circ} \mathrm{C}$ for $30 \mathrm{sec}$, and $95^{\circ} \mathrm{C}$ for $15 \mathrm{sec}$. The primer sets for the detected 
genes are listed in Table II. miRNAs were reverse transcribed by using TaqMan MicroRNA reverse transcription kit (Invitrogen; Thermo Fisher Scientific, Inc.) according to the manufacturer's instructions. TaqMan MicroRNA Assays and the TaqMan Universal PCR Master Mix (Invitrogen; Thermo Fisher Scientific, Inc.) were used to examine miRNA expression, with the thermocycling conditions as $10 \mathrm{~min}$ at $95^{\circ} \mathrm{C}$ followed by 40 cycles of $95^{\circ} \mathrm{C}$ for $15 \mathrm{sec}$ and $60^{\circ} \mathrm{C}$ for $30 \mathrm{sec}$. Data analysis was conducted by using the $2^{-\Delta \Delta \mathrm{Cq}}$ method (35), and all RT-qPCR reactions were performed in triplicate.

Dual-Luciferase reporter assay. 293T cells ( $70 \%$ confluence) were co-transfected with luciferase reporter plasmid (200 ng), miR-200c mimics/mi-NC (100 nM), and the internal control pRL-SV40 (10 ng). Cells were harvested and lysed; the luciferase activity was determined by the Dual-Luciferase Reporter Assay System (Promega Corp.) and quantified using a luminometer. The Renilla luciferase activity was used as an internal reference to adjust the deviation caused by the varying cell numbers plated and transfection efficiency.

Xenograft tumor model. All experiments were approved by the Animal Ethics Committee of Peking University Cancer Hospital \& Institute and performed in full compliance with the Experimental Animal Management Ordinance produced by the Peking University Cancer Hospital \& Institute. A total of 10 female BALB/c nude mice $(20 \mathrm{~g})$ were purchased from Hua-Fu-Kang Corp. (Beijing, China) and randomly divided into two groups. Each mouse was subcutaneously inoculated with $2 \times 10^{6}$ of the indicated cells into the right forelimb armpit. When the tumor mass became palpable, the tumor volume was measured by a caliper every three days and calculated using the formula: Volume $=$ length $\mathrm{x}$ width ${ }^{2} / 2$. At day 32 following inoculation, the mice were sacrificed, and the xenografts were extracted, imaged and processed for further analysis.

Bioinformatics analysis. The differential expression of KIF14 mRNA between cancerous and noncancerous CRC tissues was analyzed by using two integrated data-mining platforms, Gene Expression across Normal and Tumor tissue (GENT) (36) and Oncomine (37), which contain a collection of rearranged substantial microarray data for cancer profiling. Data from three datasets GSE8671, GSE21510 and GSE20916 were derived from GENT. To reduce the false discovery rate, $\mathrm{P}<0.01$ was set as a threshold for Oncomine data mining.

Gene set enrichment analysis (GSEA) was conducted by using the GSEA-2.2.3 software package (Broad Institute, Cambridge, MA, USA) $(38,39)$ to identify the genes biologically associated with CRC. The cBioPortal for Cancer Genomics (40) was used to investigate the genetic alterations of KIF14. Information regarding KIF14 gene mutations, copynumber alterations (CNAs), and abnormal mRNA expression levels (RNA-Seq V2 RSEM) was obtained from the provisional The Cancer Genome Atlas (TCGA) CRC dataset with 629 samples available at present. Transcript values were transformed into $\log 2$ values, and CNAs were determined with GISTIC 2.0 (41). miRNA target prediction algorithms, including microRNA.org (http://www.microrna.org) (42) and TargetScan (http://www.targetscan.org) (43), were applied to predict the potential miRNAs that target the KIF14 3'UTR.
Statistical analysis. All experiments were conducted no less than three times with each sample tested in triplicate. Data are presented as the mean \pm standard deviation. A two-tailed Student's t-test or a Mann-Whitney U-test was employed to evaluate the difference between two groups where appropriate. One-way analysis of variance followed by a post hoc Dunnett's test was used to determine statistical differences for multiple comparisons. Pearson correlation analysis was conducted to assess the correlation between two genes of interest. Statistical analysis was performed using SPSS software 20.0 (IBM Corp, Armonk, NY, USA) and GraphPad Prism 5 software (GraphPad Software, Inc., La Jolla, CA, USA). P<0.05 was considered to indicate a statistically significant difference.

\section{Results}

KIF14 is significantly upregulated in CRC. To assess whether KIF14 was ectopically overexpressed in CRC, integrated microarray data from GENT (36) and Oncomine (37) were analyzed in the present study. A total of three Gene Expression Omnibus (GEO) datasets, including GSE8671, GSE21510 and GSE20916 were profiled with Affymetrix U133 Plus 2.0 platforms; the gene expression values of the CRC and corresponding normal tissues were selected from GENT. In each dataset, KIF14 was significantly overexpressed in CRC specimens compared with in the corresponding normal samples (Fig. 1A). In addition, KIF14 expression levels were significantly correlated with the mRNA levels of proliferation-related Ki-67 antigen (MKI67), a well-established proliferation marker of CRC (44), in all examined datasets (Fig. 1B). Consistent with the findings of analysis with GENT, elevated KIF14 expression levels were also observed in CRC samples compared with the benign tissues from 14 analyses with the Oncomine database (Fig. 1C). Furthermore, KIF14 expression was detected in 36 pairs of fresh frozen CRC specimens by RT-qPCR. In addition to the results from bioinformatics analyses, $63.9 \%(23 / 36)$ of cases exhibited significant upregulation of KIF14 in CRC compared with in adjacent noncancerous tissues (Fig. 1D). Increased KIF14 expression levels were significantly associated with tumor size $(\mathrm{P}=0.008)$ and MKI67 immunostaining scores $(\mathrm{P}=0.016$; Table $\mathrm{I})$. In addition, the mRNA and protein expression levels of KIF14 were detected in the five CRC cell lines by RT-qPCR and western blotting (Fig. 1E and F). The results revealed that KIF14 was upregulated in SW620, SW480 and RKO cells, and downregulated in HCT116 and LoVo cells.

KIF14 accelerates $C R C$ cell proliferation by modulating progression of the cell cycle. To investigate the function of KIF14 in the progression of CRC, specific siRNA oligos targeting KIF14 were introduced into SW480 and RKO cells. Additionally, a recombinant expression plasmid encoding KIF14 was constructed and transfected into LoVo cells to observe alterations in phenotype associated with KIF14. The transfection efficiency of KIF14 siRNA or its overexpression vector was confirmed by RT-qPCR and western blotting in SW480 or LoVo cells (Fig. 2A and B). By using an MTT assay, the present study demonstrated that decreased KIF14 expression resulted in markedly reduced cell viability of SW480 and RKO cells, and upregulated KIF14 promoted the proliferation of LoVo cells (Fig. 2C). In addition, the colony formation assay 

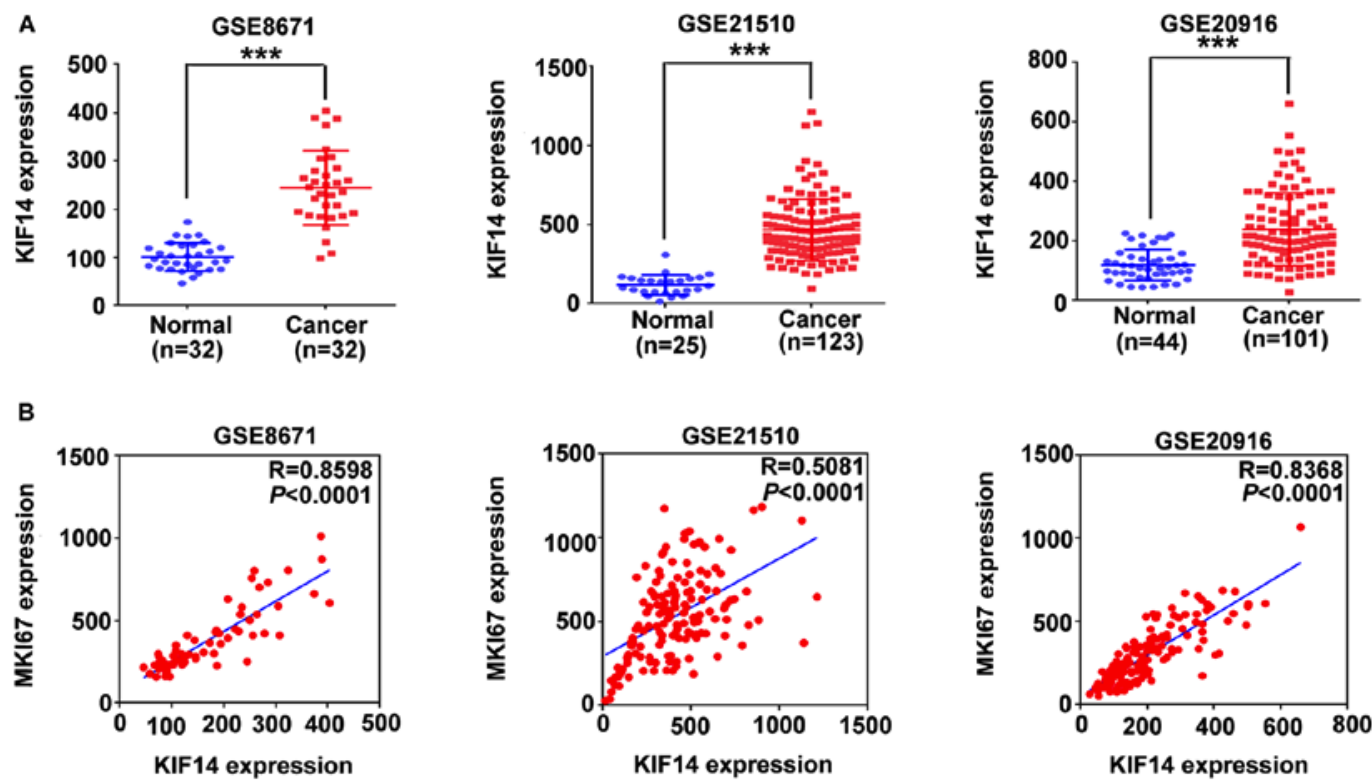

C

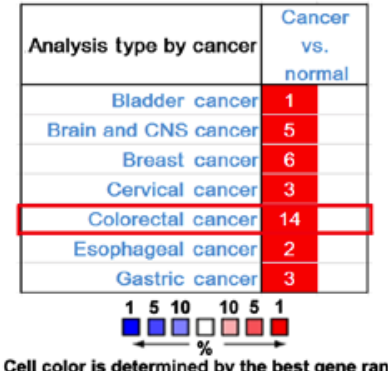
percentile for the analyses within the cell
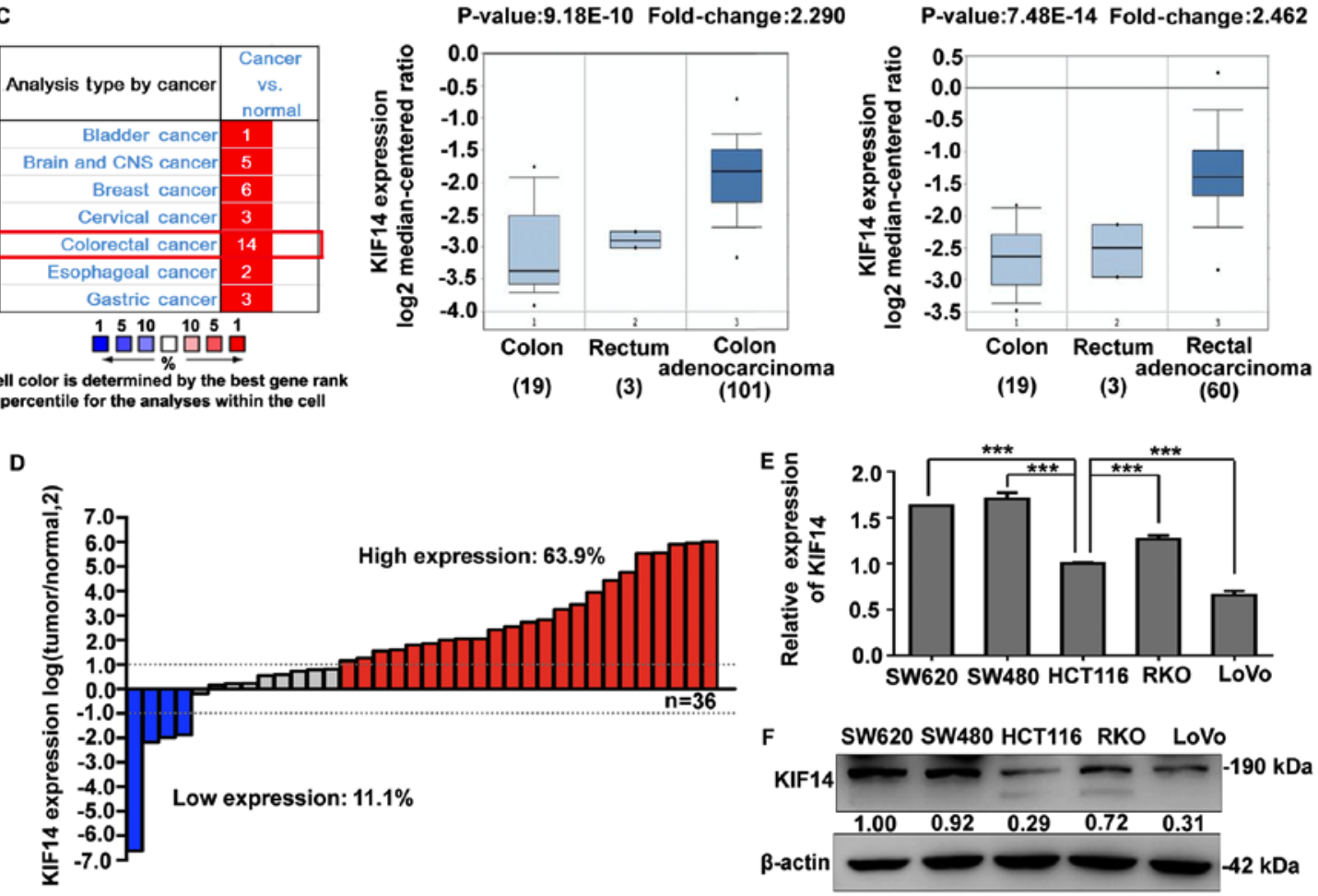

Figure 1. Expression of KIF14 in CRC specimens and cell lines. (A) KIF14 expression was analyzed in the GSE8671, GSE21510 and GSE20916 CRC datasets from the Gene Expression across Normal and Tumor tissue database. (B) Correlation between KIF14 and MKI67 was investigated via Pearson's correlation analysis. (C) Left panel, summarization of KIF14 expression in various cancer types from the Oncomine database. Middle and right panels, KIF14 mRNA was overexpressed in colon and rectal adenocarcinomas compared with normal epithelia. (D) KIF14 mRNA expression in 36 pairs of fresh frozen CRC tissues was determined by RT-qPCR. Different colors of the bars indicate the proportions of CRC samples with upregulated (red, $>2$-fold), downregulated (blue, $<2$-fold) and unchanged (gray) expression of KIF14. (E and F) mRNA and protein expression levels of KIF14 were evaluated by RT-qPCR and western blotting in five CRC cell lines. The results were presented as the mean \pm standard deviation from three independent experiments, ${ }^{* * *} \mathrm{P}<0.001$. KIF 14 , kinesin family member 14; CRC, colorectal cancer; MKI67, proliferation-related Ki-67 antigen; RT-qPCR, reverse transcription-quantitative polymerase chain reaction.

produced similar results to that of the MTT assay (Fig. 2D). Furthermore, the DNA synthesis of treated CRC cells was examined by an EdU incorporation assay. As presented in Fig. 2E, transfection of KIF14 siRNA attenuated the ratio of EdU-positive SW480 and RKO cells, whereas overexpressed KIF14 significantly accelerated the DNA synthesis of LoVo cells.
To understand the potential mechanism by which KIF14 promotes CRC cell proliferation, flow cytometry was conducted to investigate the effects of KIF14 on the cell cycle of CRC. Depletion of KIF14 increased the G1 phase population of SW480 and RKO cells, and reduced that of the $\mathrm{S}$ and $\mathrm{G} 2$ phase population. Additionally, overexpressed KIF14 promoted the transition of LoVo cells in G1 to enter 

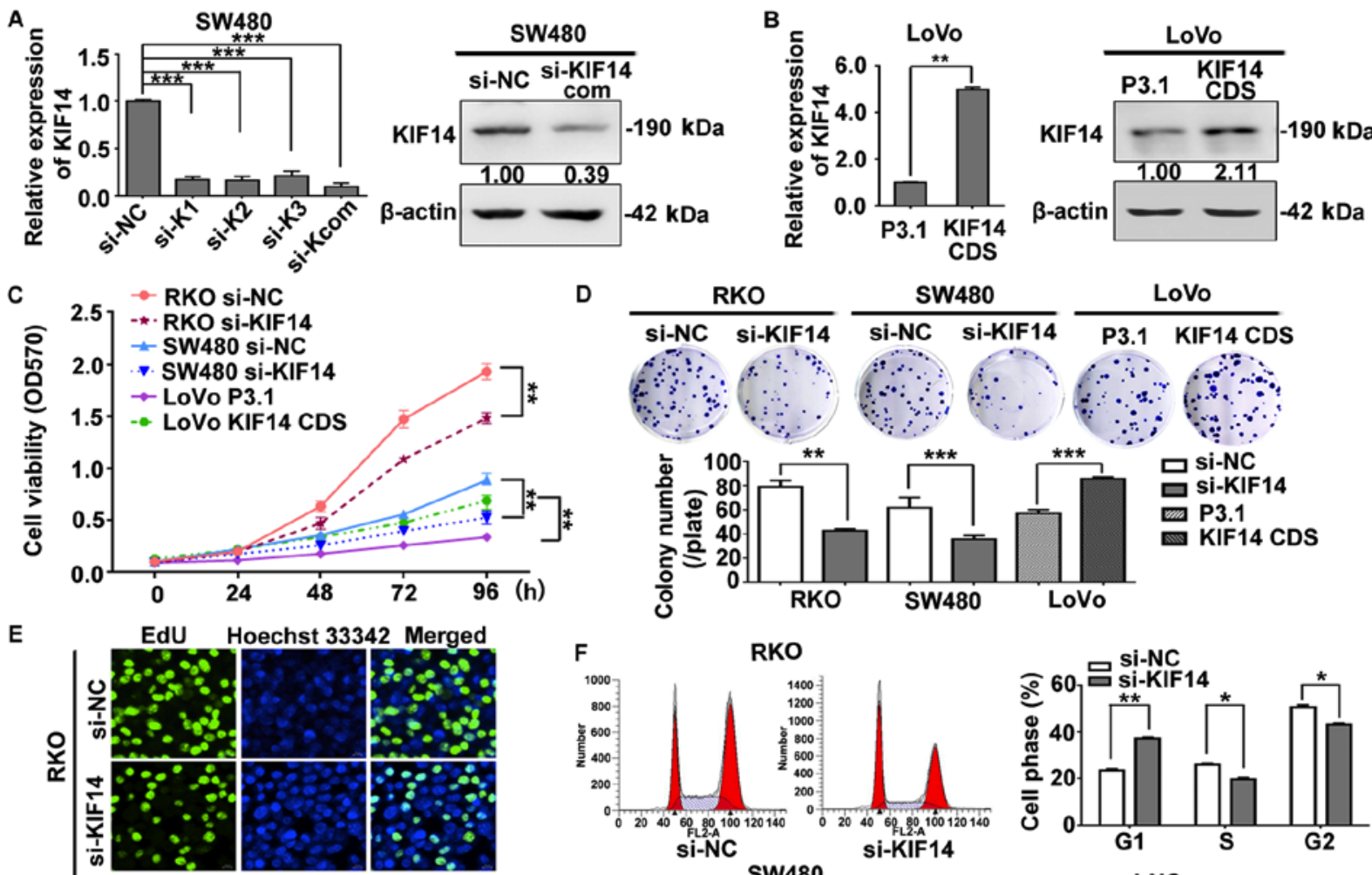

D RKO

$-190 \mathrm{kDa}$ $-42 \mathrm{kDa}$
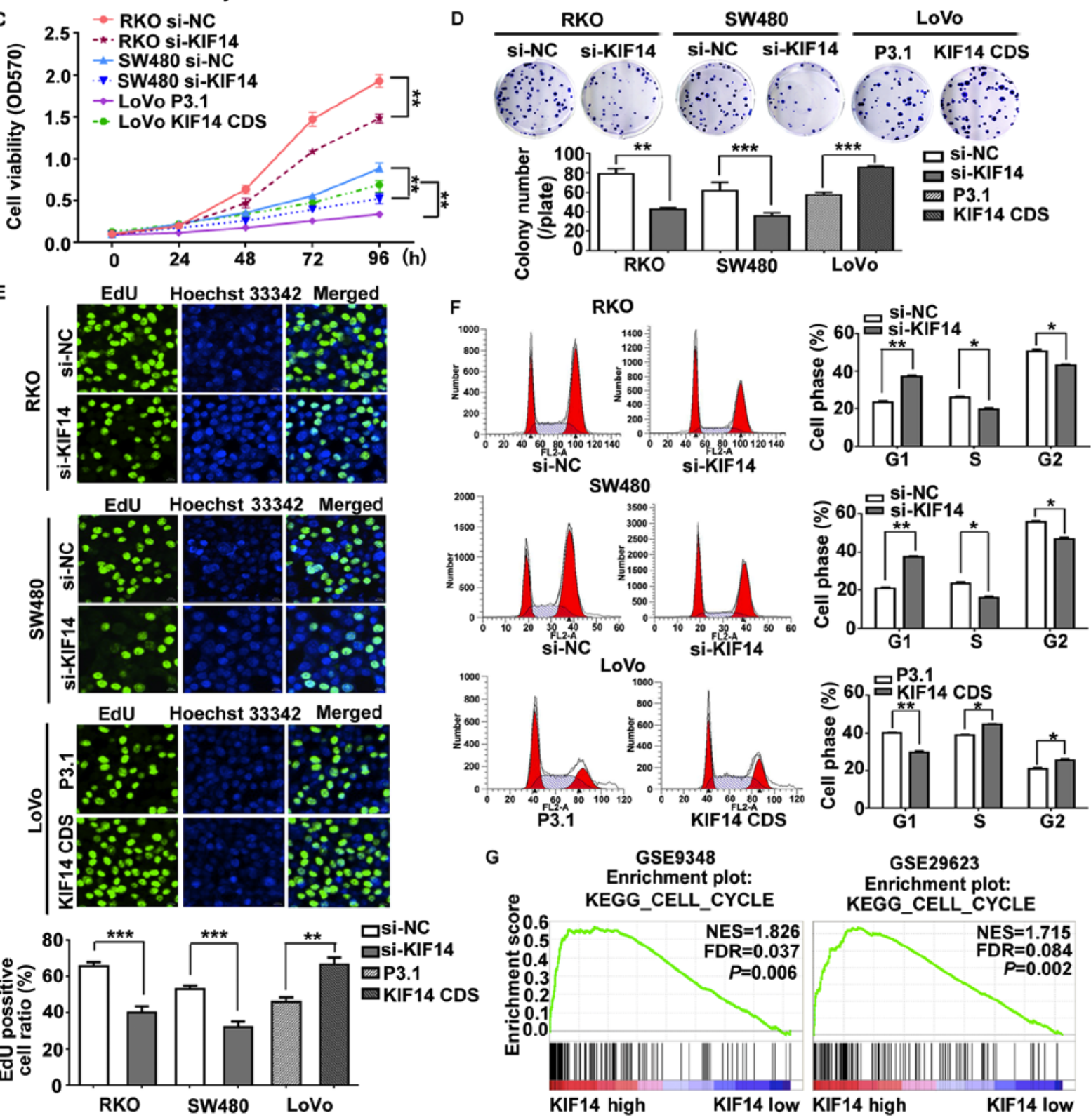

GSE29623

Enrichment plot: KEGG_CELL_CYCLE

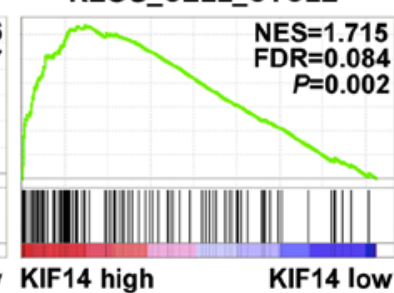

Figure 2. KIF14 promotes CRC cell proliferation and accelerates the cell cycle. (A) SW480 or (B) LoVo cells were transfected with three KIF14 siRNA oligos or the siRNA complex and the P3.1-KIF14 CDS plasmid, respectively, and their corresponding negative controls; the mRNA and protein expression levels of KIF14 were examined by reverse transcription-quantitative polymerase chain reaction and western blotting. (C) Cell proliferation of SW480 and RKO cells transfected with KIF14 siRNA or NC siRNA, and LoVo cells transfected with P3.1-KIF14 CDS or the control vector, was detected via a $96 \mathrm{~h}$ MTT assay. (D) Cell viability of CRC cells, which were treated as aforementioned, was determined via a colony formation assay. (E) DNA synthesis was analyzed in CRC cells transfected with the corresponding siRNA or plasmids via an EdU incorporation assay (scale bar, $20 \mu \mathrm{m}$ ). (F) Distribution of the cell cycle was examined by flow cytometry in silenced KIF14 SW480 and RKO cells, or LoVo cells exhibiting KIF14 overexpression, in addition to the corresponding controls. The distribution of the cell population of different treatment groups was presented as a bar graph. (G) Enrichment plots of gene expression signatures associated with the cell cycle in the GSE9348 and GSE29623 datasets according to KIF14 mRNA expression levels. All results were presented as the mean \pm standard deviation ( $\mathrm{n}=3),{ }^{*} \mathrm{P}<0.05,{ }^{* *} \mathrm{P}<0.01,{ }^{* * *} \mathrm{P}<0.001$. CRC, colorectal cancer; KIF14, kinesin family member 14; si, small interfering RNA; NC, negative control; EdU, 5-ethynyl-2-deoxyuridine; FDR, false detection rate; NES, normalized enrichment score;

into $\mathrm{S}$ and $\mathrm{G} 2$ phase compared with the control (Fig. 2F). In addition, the results from GSEA analysis (38) with two GEO datasets of CRC revealed that the cell cycle-associated gene set was significantly enriched in KIF14 overexpressed cases, 

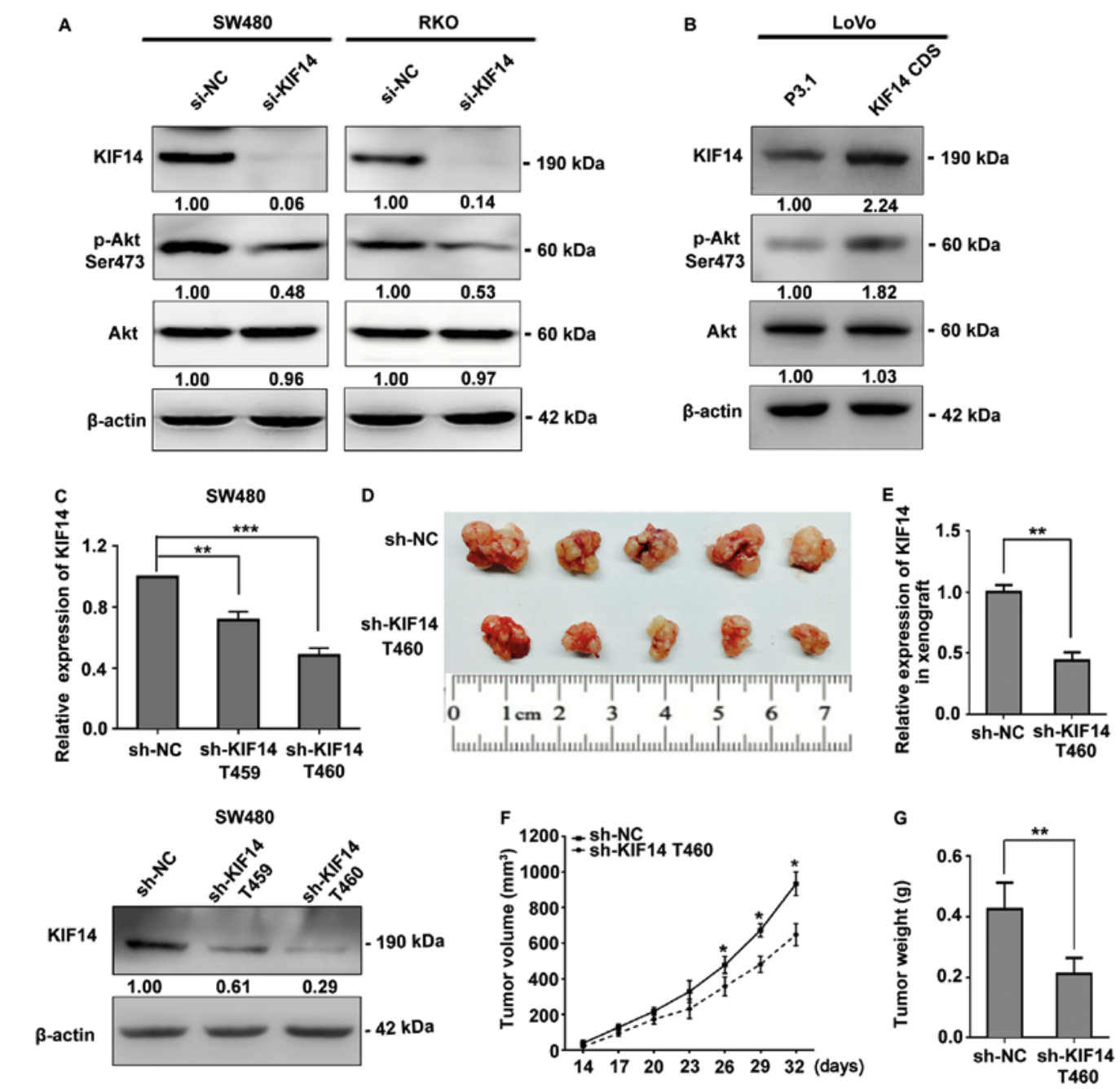

Figure 3. KIF14 activates Akt signaling and promotes CRC tumorigenesis in vivo. Alterations in Akt phosphorylation activity due to (A) attenuated or (B) upregulated KIF14 expression in CRC cells were determined by western blotting. $\beta$-actin served as an internal control. (C) Transduction efficiency of recombinant lentiviruses KIF14 shRNA was determined by RT-qPCR and western blotting. All values were presented as the mean \pm standard deviation $(\mathrm{n}=3)$. (D) Resected subcutaneous tumors generated by SW480 cells stably transduced with KIF14 shRNA T460 or the control (n=5). (E) KIF14 expression in xenografts at day 32 was measured by RT-qPCR. (F) Tumor volume was recorded seven times at equal intervals from the 14th day following injection. (G) Weight of resected xenografts was measured. The results from the xenograft tumor model were presented as the mean \pm standard deviation $(\mathrm{n}=5)$. ${ }^{* *} \mathrm{P}<0.01$, ${ }_{* * * * *} \mathrm{P}<0.001$. CRC, colorectal cancer; RT-qPCR, reverse transcription-quantitative polymerase chain reaction; si, small interfering RNA; NC, negative control; KIF14, kinesin family member 14; p-, phospho-; Akt, protein kinase B; sh, short hairpin RNA.

supporting that KIF14 participates in the regulation of the cell cycle (Fig. 2G).

KIF14 activates Akt signaling and promotes CRC tumorigenesis in vivo. It has been reported that Akt activation is necessary for cell cycle progression $(45,46)$. To determine whether KIF14 activated Akt in CRC, thus accelerating the progression of the cell cycle, western blotting was performed. As presented in Fig. 3A, silencing of KIF14 in SW480 and RKO cells resulted in notably decreased phosphorylation of Akt. Furthermore, upregulation of KIF14 in LoVo cells led to markedly increased phosphorylation of Akt (Fig. 3B).

To determine whether KIF14 promoted CRC tumor progression in vivo as observed in vitro, a tumorigenicity assay with nude mice was performed. Two recombinant lentiviruses containing shRNA targeting KIF14 (KIF14 shRNA T459 and T460) or a control were transduced into SW480 cells, respectively. Subsequently, the cells were selected with puromycin at $2 \mu \mathrm{g} / \mathrm{ml}$ for a week. Following detection via RT-qPCR and western blotting, the subgroup of cells stably expressing KIF14 shRNA T460 was selected for further analysis due to its higher efficiency knockdown of KIF14 compared with those without stable expression (Fig. 3C). Thereafter, SW480 cells containing KIF14 shRNA T460 or the control shRNA were subcutaneously inoculated into the right forelimb armpit of nude mice. The tumor volume was measured every 3 days, starting at day 14 after injection. The mice were sacrificed on day 32 for tumor weight analysis. The tumor size of the nude mice injected with KIF14 shRNA T460-containing cells was notably smaller than that of the control group (Fig. 3D). Additionally, KIF14 expression was markedly decreased in the experimental group as determined by RT-qPCR (Fig. 3E). The tumor volume was significantly decreased in the KIF14 knockdown group for at least three measurements compared with in the control (Fig. 3F). In accordance with this, the tumor weight was significantly 
A

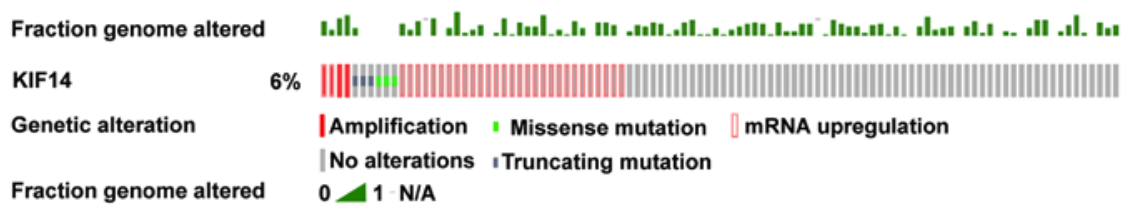

B
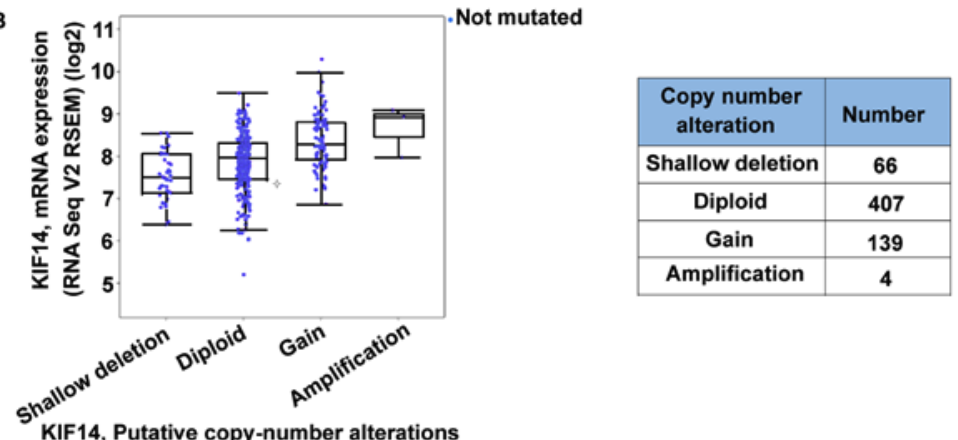

C GSE21510
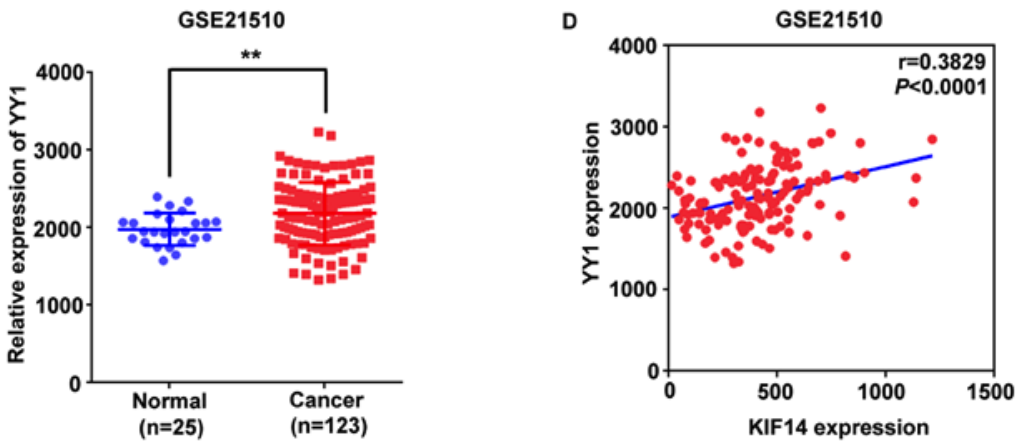

Figure 4. Genetic alterations and transcriptional regulation of KIF14 in human CRC. (A) Oncoprint plot of The Cancer Genome Atlas provisional CRC dataset generated by cBioPortal revealed KIF14 genetic alterations in a total of 629 patients analyzed. (B) mRNA expression of KIF14 (RNAseq V2 RSEM) was plotted against the putative copy-number alterations from GISTIC. (C) YY1 was upregulated in cancer tissues compared with in normal counterparts in the GSE21510 CRC dataset from the Gene Expression across Normal and Tumor tissue database. (D) Correlation between YY1 and KIF14 expression was determined by Pearson's correlation analysis. ${ }^{* *} \mathrm{P}<0.01$. CRC, colorectal cancer; KIF14, kinesin family member 14; YY1, YY1 transcription factor.

lower in the KIF14 shRNA T460-transfected cell group compared with in the control group (Fig. 3G).

Genomic amplification and transcriptional regulation may lead to KIF14 overexpression in CRC. To investigate the mechanism underlying the overexpression of KIF14 in CRC at the genetic level, analysis was performed using the cBioPortal for Cancer Genomics (40). A previous study reported that certain patients with CRC exhibited a chromosomal gain of 1q31-1q32 at the location of the KIF14 coding gene (32), and in silico screening of KIF14 in TCGA provisional CRC dataset further supported this finding. As demonstrated in Fig. 4A, 6\% cases (39/629) presented at least one genetic alteration of KIF14. Additionally, KIF14 mRNA expression levels gradually increased, along with putative KIF14 CNAs (in ascending order as shallow deletion, diploid, gain and amplification), indicating that genomic amplifications may partially contribute to abnormally overexpressed KIF14 in CRC (Fig. 4B). Additionally, the phenomenon that certainpatients with upregulated KIF14 present a notable unchanged fraction of genomic alterations (Fig. 4A) suggested that KIF14 overexpression may not be limited to the genetic level. In addition, the expression of transcription factor YY1, which regulates KIF14 (47), was increased in CRC samples and was also positively correlated with the expression of KIF14 in the GSE21510 dataset (Fig 4C and D). This indicated that upregulated expression levels of YY1 may activate KIF14 transcription, leading to KIF14 overexpression.

KIF14 is a direct target of miR-200c. Whether KIF14 is regulated at the post-transcriptional level in CRC remains unknown. To identify the potential miRNAs targeting KIF14, two online bioinformatics tools, microRNA.org (42) and TargetScan (43), were used, and the prediction results were comprehensively analyzed. The candidate miRNAs were sorted and ranked according to the total mirSVR scores. Analysis revealed that three mature miRNAs from the miR-200 family, including miR-200c, miR-200b and miR-429, exhibited the greatest potential to regulate KIF14. To validate the findings of the present study, the endogenous expression of miR-200c, miR200b and miR-429 was detected in the CRC cell lines (Fig. 5A). To identify the specific miRNA that regulated KIF14 with the highest potency, the expression of these three miR-200 family members were investigated in KIF14-silenced CRC cells. As presented in Fig. 5B, knockdown of KIF14 led to notably increased miR-200c expression levels in SW480 and RKO cells; miR-200b and miR-429 were upregulated in RKO cells following KIF14 knockdown. These observations indicated 


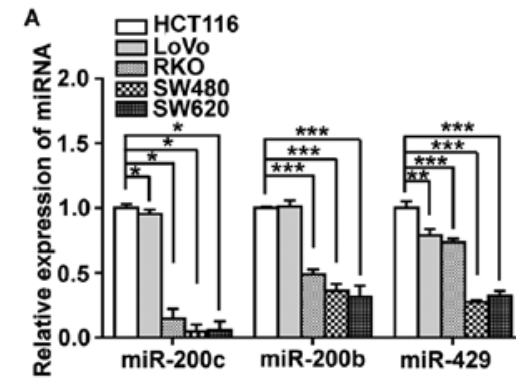

C
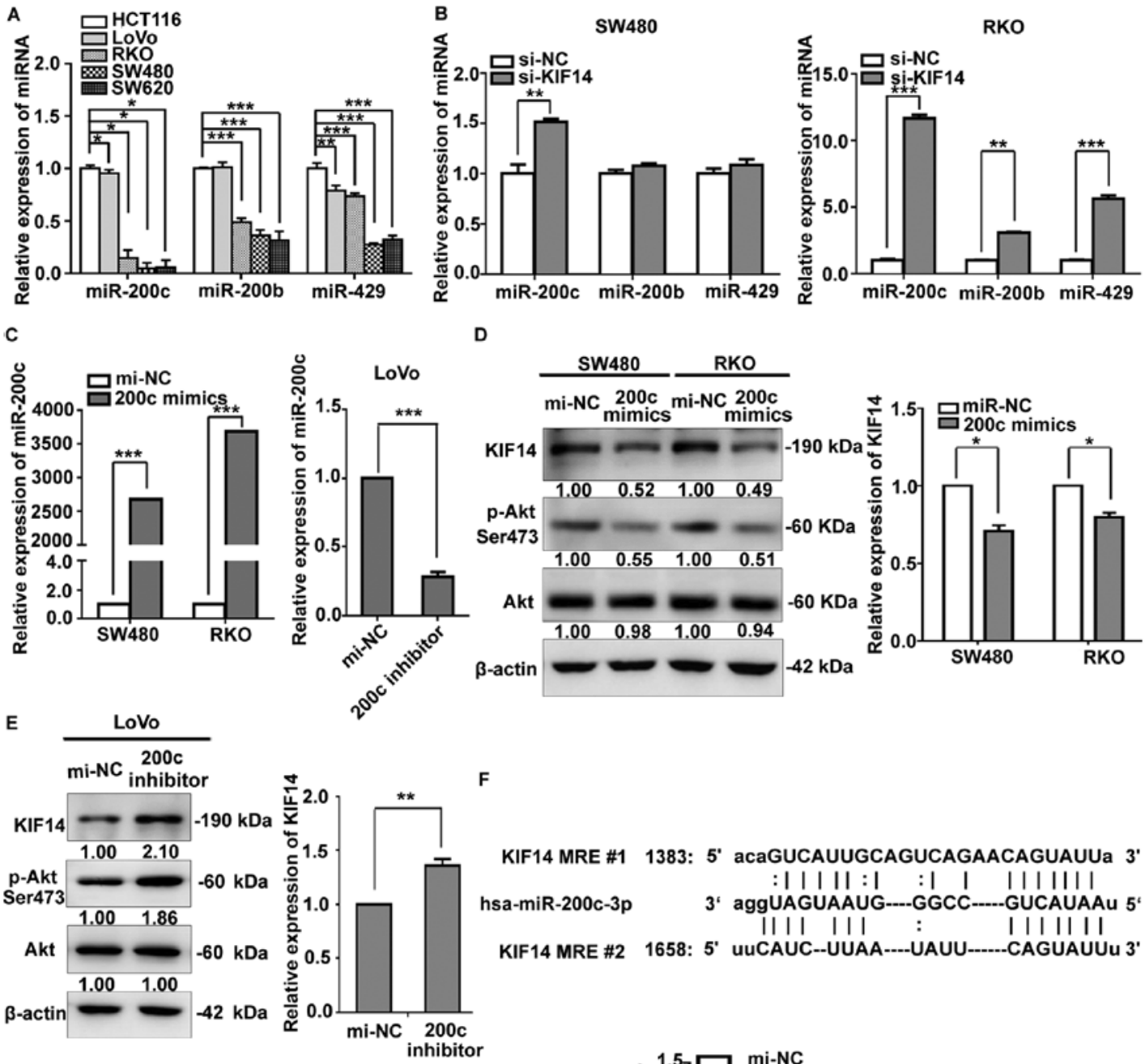

$\mathbf{F}$

KIF14 MRE \#1 1383: 5' acagucAUUgCAguCAgaACAgUAUUa $3^{\circ}$ :|||||:| :| | |||||||

III ||| : II| III|

KIF14 MRE \#2 1658: 5' UuCAUC--UUAA-..--UAUU---..-CAGUAUUu 3'

G
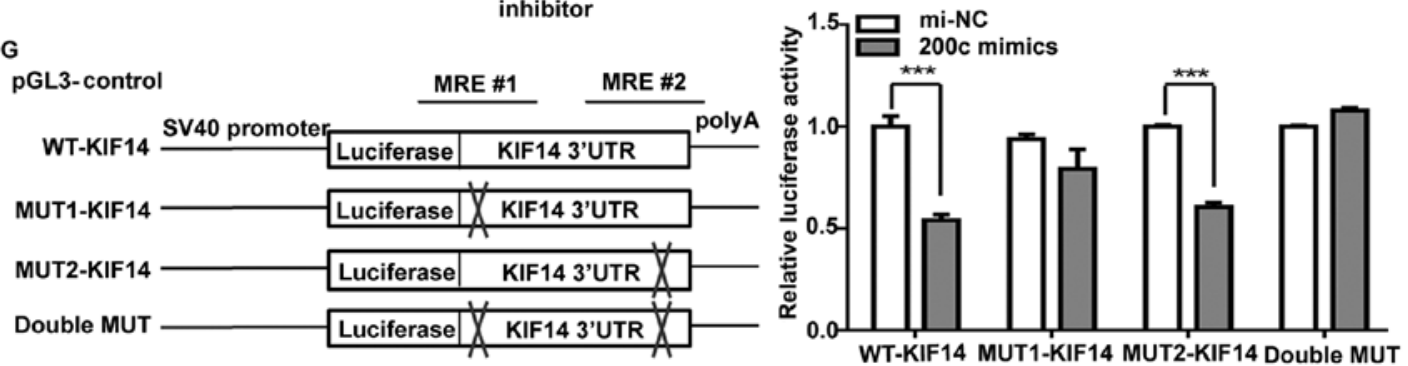

Figure 5. miR-200c modulates KIF14 expression most potently. (A) miR-200c, miR-200b and miR-429 expression levels were detected in five colorectal cancer cell lines by RT-qPCR. (B) Expression of miR-200c, miR-200b and miR-429 was measured by RT-qPCR in SW480 and RKO cells transfected with KIF14 siRNA or the control. (C) miR-200c mimics or inhibitor was transfected into SW480, RKO or LoVo cells respectively, and the transfection efficiency was determined by RT-qPCR. Effects of miR-200c (D) mimics or (E) inhibitor on KIF14 protein expression levels and the phosphorylation levels of Akt were examined by western blotting. $\beta$-actin served as an internal control. RT-qPCR was used to detect the alteration of KIF14 mRNA expression. (F) Schematic diagram of miR-200c binding sites in the 3'UTR of KIF14 from microRNA.org. (G) Left panel, construction of KIF14 luciferase reporter plasmids harboring the wild-type, single mutated or double mutated miR-200c response element. Right panel, the relative luciferase activity was measured in HEK293 cells co-transfected with reporter plasmids containing the wild-type or mutant KIF14 3'UTR sequences and miR-200c mimics/mi-NC. All data were expressed as the mean \pm standard deviation $(\mathrm{n}=3) .{ }^{*} \mathrm{P}<0.05,{ }^{* *} \mathrm{P}<0.01,{ }^{* * *} \mathrm{P}<0.001$. RT-qPCR, reverse transcription-quantitative polymerase chain reaction; UTR, untranslated region; miR, microRNA; si, small interfering RNA; NC, negative control; KIF14, kinesin family member 14; mi-NC, negative control microRNA; MRE, microRNA response element; WT, wild-type; MUT, mutant.

that miR-200c bound to and modulated KIF14 expression in both SW480 and RKO cells; reduced KIF14 mRNA released more miR-200c and induced elevated expression levels of miR-200c. While the regulation of KIF14 by miR-200b and miR-429 may be cell type-specific. The present study found that miR-200c regulated KIF14 most effectively.

To validate the findings of the present study, miR-200c mimics transfected into SW480 and RKO, and inhibitor was transfected into LoVo cells, in addition to a control. Following confirmation of the transfection efficiency (Fig. 5C), western blotting and RT-qPCR were conducted to examine whether KIF14 was modulated by miR-200c. The results demonstrated that upregulated miR-200c led to decreased expression of KIF14 at the protein and mRNA levels in SW480 and RKO cells, and also inhibited Akt activity (Fig. 5D). Additionally, miR-200c inhibitor elevated the protein expression level of 


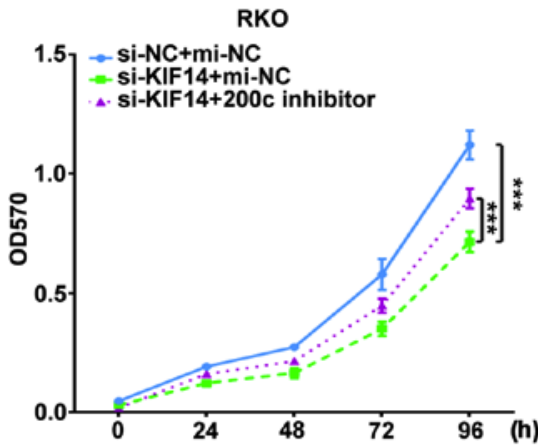

si-NC+mi-NC siKIF14+mi-NC si-KIF14+200c inhibitor
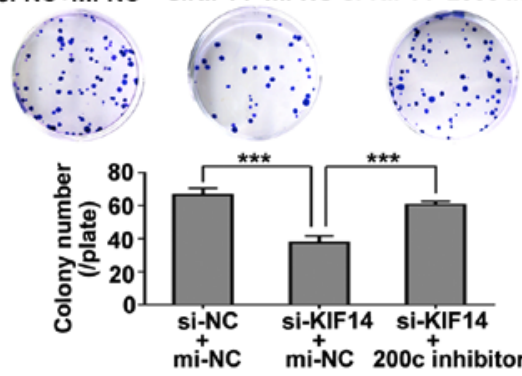

C RKO

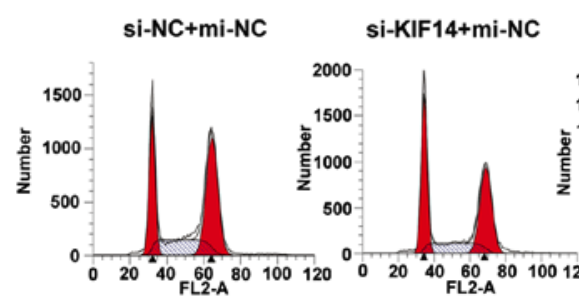

si-KIF14+200c inhibitor

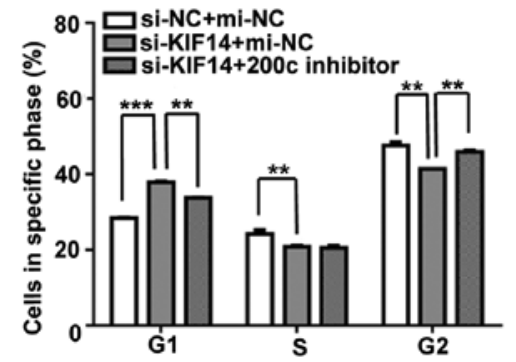

D
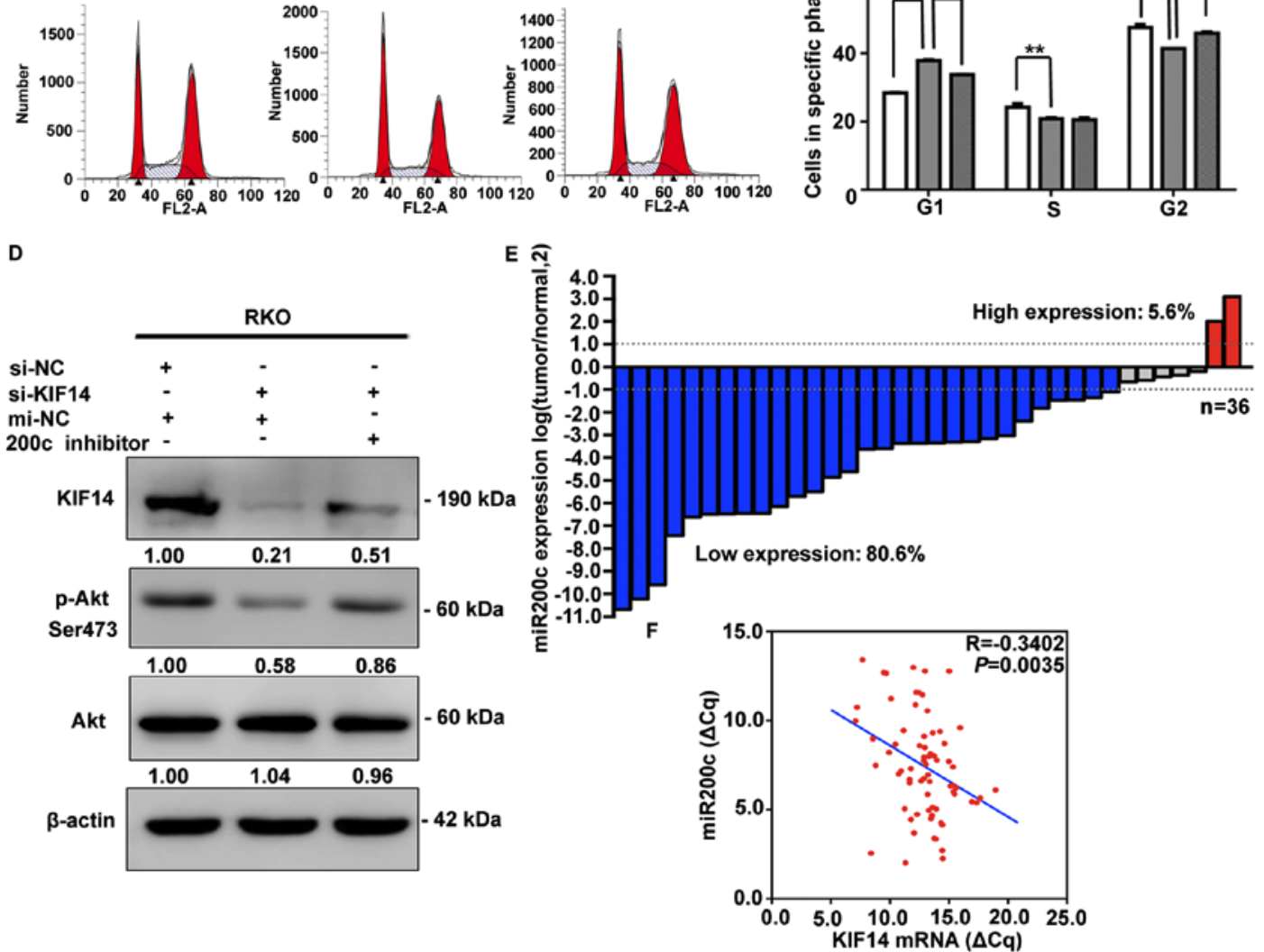

Figure 6. miR-200c participates in the regulation of CRC cell proliferation by targeting KIF14 and is negatively correlated with KIF14 in vivo. Reduced cell viability of RKO cells due to KIF14 siRNA transfection was partly restored following the silencing of miR-200c, which was detected by (A) MTT and (B) colony formation assays. (C) Cell cycle was analyzed by flow cytometry when RKO cells were co-transfected with KIF14 siRNA and miR-200c inhibitor, or their corresponding controls. (D) Inhibition of miR-200c elevated the protein expression levels of KIF14 and the phosphorylation levels of Akt, which was downregulated by KIF14 siRNA previously. (E) miR-200c expression was evaluated in 36 paired primary CRC and adjacent normal tissues by reverse transcription-quantitative polymerase chain reaction. The various colors of the bars indicated the proportions of CRC samples with upregulated (red, $>2$-fold), downregulated (blue, $<2$-fold) and unchanged (gray) expression of miR-200c. (F) Correlation between miR-200c and KIF14 in these specimens (DCq values were normalized to GAPDH) was determined via Pearson's correlation analysis. All data were expressed as the mean \pm standard deviation ( $\mathrm{n}=3$ ), ${ }^{* *} \mathrm{P}<0.01$, ${ }^{* * * *} \mathrm{P}<0.001$. CRC, colorectal cancer; miR, microRNA; si, small interfering RNA; mi-NC, negative control microRNA; KIF14, kinesin family member 14; p-, phospho-; Akt, protein kinase B.

KIF14 and the Ser473 phosphorylation of Akt in LoVo cells (Fig. 5E).

According to the bioinformatics analysis, two miR-200c response elements were observed to be located at 1,4021,409 bp and 1,672-1,678 bp of the KIF14 3'UTR (Fig. 5F). To determine whether miR-200c directly targeted KIF14 via these two binding sites, WT KIF14 3'UTR fragment or mutated sequences with a MUT1/MUT2 or DOU MUT miR-200c response element deletion were inserted following the coding sequence of the pGL3-Control reporter construct (Fig. 5G). 293T cells were co-transfected with either WT or MUT KIF14 3'UTR reporter plasmids, along with miR-200c mimics or the corresponding control, respectively. miR-200c directly reduced the luciferase activity of pGL3-WT KIF14 3'UTR compared with in the control (Fig. 5G). Similarly, attenuated luciferase activities of pGL3-MUT2 KIF14 3'UTR were detected when cells were co-transfected with miR-200c mimics other than the control oligo (Fig. 5G). However, the 
KIF14 3'UTR reporter constructs with the first miR-200c binding site (MUT-1) or both miR-200c binding sites (DOU MUT) deleted were unaffected by miR-200c mimics (Fig. 5G). Therefore, KIF14 was proposed as a direct target of miR-200c; the 1,402-1,409 bp locus of KIF14 may be the essential binding site for miR-200c.

miR-200c is involved in KIF14-mediated CRC cell proliferation and is negatively correlated with KIF14 in vivo. To confirm whether KIF14 was a functional target of miR-200c, rescue experiments were performed in RKO cells. The growth-inhibitory effect caused by KIF14 knockdown was partly reversed via miR-200c silencing as demonstrated by MTT and colony formation assays (Fig. 6A and B). Cell cycle analysis revealed that RKO cells accumulated in G1 phase due to downregulated KIF14 partially transitioned into S/G2 phase following co-transfection with miR-200c inhibitor (Fig. 6C). Furthermore, co-transfection of miR-200c inhibitor restored the decreased protein expression levels of KIF14 mediated by siRNA silencing, and elevated the reduced phosphorylation levels of Akt (Fig. 6D). These findings suggested that miR-200c modulated the function of KIF14, and could influence CRC cell survival by regulating KIF14.

In addition, the expression of miR-200c in 36 pairs of CRC specimens was determined by RT-qPCR. The majority of the CRC specimens $(80.6 \%)$ presented significantly decreased expression levels of miR-200c compared with in the adjacent normal tissues (Fig. 6E). Additionally, a significant inverse correlation was observed between the expression of miR-200c and KIF14 (Pearson R=-0.3402; P=0.0035) (Fig. 6F), which suggested that KIF14 was regulated by miR-200c in vivo.

\section{Discussion}

Accumulating evidence has demonstrated that KIF14 is involved in the initiation and progression of a variety of human cancers (23-31); however, the biological function and clinical significance of KIF14 in colorectal tumorigenesis remain poorly understood. In the present study, KIF14 was observed to be abundantly overexpressed in CRC tissues and associated with proliferative indicators. Functional investigations demonstrated that KIF14 promoted tumor proliferation via regulation of the cell cycle and activation of Akt signaling. In addition, the present study reported that downregulated miR-200c may partly contribute to KIF14 overexpression and facilitate KIF14 to exert its oncogenic role in CRC.

Previous studies have revealed that KIF14 is a robust prognostic indicator in numerous cancers $(24,28,31)$. Significant upregulation of KIF14 was observed in CRC samples; however, KIF14 expression was only correlated with the indicators associated with proliferation, which may be due to the limited sample size in the present study. To further validate the correlation between KIF14 and the proliferative index MKI67, the expression levels of KIF14 and MKI67 were investigated using publicly available datasets. A strong correlation was observed between KIF14 and MKI67 mRNA expression levels in the datasets employed, which further demonstrated the pro-proliferative characteristics of KIF14. These findings suggested that KIF14 may be a potential novel marker of proliferation and therapeutic target for CRC.
In vitro and in vivo experiments confirmed the correlation between KIF14 overexpression and CRC cell proliferation. The altered ratio of EdU incorporation along with altered KIF14 expression levels further indicated that KIF14 was involved in the cell cycle progression of CRC. Consistently, the results from flow cytometric analysis revealed that depletion of KIF14 may induce G1 arrest in RKO and SW480 cells, while overexpressed KIF14 accelerated the cell cycle of LoVo cells. Additionally, GSEA analysis demonstrated the promoting effect of KIF14 on cell cycle.

As a mitotic kinesin, KIF14 has been reported to be crucial for midbody formation and the completion of cytokinesis during telophase (19). Carleton et al (19) documented that notable reduction in the expression levels of KIF14 prevented midbody cleavage and induced cytokinesis failure; less efficacious KIF14 knockdown resulted in acute apoptosis at certain points in the cell cycle (19). Based on the aforementioned findings, the KIF14 siRNAs employed in the present study were suggested to lead to cell division failure and multinucleation; thus the progression of the cell cycle of non-viable daughter cells may be inhibited and cells will not transition into $\mathrm{S}$ phase. Apart from interfering with cytokinesis, downregulated KIF14 may also inhibit the cell cycle by suppressing the ubiquitination of cyclin-dependent kinase inhibitor $1 \mathrm{~B}$ in hepatocellular carcinoma (25), which further demonstrates the complex functions of KIF14 in a variety of tumors. In addition, Ser473 phosphorylation of Akt induced by the overexpression of KIF14 in the present study suggested that KIF14 promoted cell survival via activation of the Akt signaling pathway, which was crucial for the progression of the cell cycle $(45,46)$.

A previous study reported that KIF14 was overexpressed in ovarian cancer via transcriptional and epigenetic regulation (47). In the present study, bioinformatics analysis revealed that abnormally overexpressed KIF14 in CRC was partially due to genomic amplification and transcriptional activation. The relatively lower proportion of KIF14 genetic alterations and the median correlation coefficient between KIF14 and YY1 may not explain the overexpression of KIF14 in CRC entirely. Therefore, the present study investigated the possible mechanism underlying the modulation of KIF14 at the posttranscriptional level to further improve understanding of the KIF14 regulatory mechanism.

Following in silico screening and validation experiments, miR-200c was reported to directly regulate the expression of KIF14. Rescue experiments were conducted to further validate whether KIF14 was a functional target of miR-200c in the present study. In addition, decreased miR-200c expression levels were inversely correlated with KIF14 expression levels within CRC tissues. A previous study revealed that miR-200c exerted antitumor effects by inhibiting metastasis and improving chemosensitivity in CRC (48); other studies also reported that the expression of $\mathrm{miR}-200 \mathrm{c}$ was significantly decreased in CRC compared with in normal tissues $(49,50)$. However, several studies have demonstrated that miR-200c was overexpressed in primary CRC lesions and gradually decreased with increasing tumor stage $(51,52)$. The factors contributing to the regulation of miR-200c expression are also complex. Hypomethylation of the miR-200c promoter regions induces miR-200c upregulation (53), while the aberrantly 
overexpressed transcriptional factors achaete-schute complex homolog 2 and Nanog suppresses the transcription of miR-200c in CRC $(49,54)$, indicating that expression of miR-200c is regulated by epigenetic alterations and transcriptional regulation. Therefore, we hypothesized that within CRC tissues exhibiting unaltered methylation of miR-200c promoter regions, decreased miR-200c expression levels were associated with KIF14 overexpression and enhanced tumor progression.

In summary, the findings of the present study, which determined the role of KIF14 and its regulatory mechanisms in CRC, may improve the current understanding of colorectal tumorigenesis and provide insight for the development of novel therapeutic agents for the treatment of CRC.

\section{Acknowledgements}

Not applicable.

\section{Funding}

The present study was supported by the grants from Beijing Municipal Natural Science Foundation (grant nos. 7172042 and 7162039), the National Natural Science Foundation of China (grant nos. 81402346, 81672439, 81272766, 81450028, 81470129 and 81502137), the Capital's Funds for Health Improvement and Research (grant no. CFH 2018-2-2153), Science Foundation of Peking University Cancer Hospital (grant no. 2017-14), Beijing Municipal Administration of Hospitals Clinical Medicine Development of Special Funding Support (grant nos. XM201309 and ZYLX201701), Beijing Health System High-level Health Technical Personnel Training Program (grant no. 2015-3-074) and the Excellent Overseas-Returnee Scholar Program (Ministry of Education).

\section{Availability of data and materials}

The datasets generated and analyzed in the present study are available at GENT (http://genome.kobic.re.kr/GENT/), Oncomine (http://www.oncomine.org), and cBioPortal for Cancer Genomics (http://www.cbioportal.org).

\section{Authors' contributions}

ZW, JY and XS conceived the experiments and drafted the manuscript. ZW and JY conducted the experiments. BJ, JD, PG and LP helped to analyze and interpret the data. All authors read and approved the final manuscript.

\section{Ethics approval and consent to participate}

Written informed consent from each patient enrolled in the study was obtained and ethical approval for the use of human tissues and for the animal study was obtained from the Ethics Committee of Peking University Cancer Hospital and Institute (Beijing, China).

\section{Consent for publication}

Not applicable.

\section{Competing interests}

The authors declare that they have no competing interests.

\section{References}

1. Torre LA, Bray F, Siegel RL, Ferlay J, Lortet-Tieulent J and Jemal A: Global cancer statistics, 2012. CA Cancer J Clin 65: 87-108, 2015

2. Favoriti P, Carbone G, Greco M, Pirozzi F, Pirozzi RE and Corcione F: Worldwide burden of colorectal cancer: A review. Updates Surg 68: 7-11, 2016.

3. Chen W, Zheng R, Zuo T, Zeng H, Zhang S and He J: National cancer incidence and mortality in China, 2012. Chin J Cancer Res 28: 1-11, 2016.

4. DeSantis CE, Lin CC, Mariotto AB, Siegel RL, Stein KD, Kramer JL, Alteri R, Robbins AS and Jemal A: Cancer treatment and survivorship statistics, 2014. CA Cancer J Clin 64: 252-271, 2014.

5. Anaya DA, Becker NS and Abraham NS: Global graying, colorectal cancer and liver metastasis: New implications for surgical management. Crit Rev Oncol Hematol 77: 100-108, 2011.

6. Stein U and Schlag PM: Clinical, biological, and molecular aspects of metastasis in colorectal cancer. Recent Results Cancer Res 176: 61-80, 2007.

7. Cancer Genome Atlas N; Cancer Genome Atlas Network: Comprehensive molecular characterization of human colon and rectal cancer. Nature 487: 330-337, 2012.

8. Uchi R, Takahashi Y, Niida A, Shimamura T, Hirata H, Sugimachi K, Sawada G, Iwaya T, Kurashige J, Shinden Y, et al: Integrated multiregional analysis proposing a new model of colorectal cancer evolution. PLoS Genet 12: e1005778, 2016.

9. Hirokawa N, Noda Y, Tanaka Y and Niwa S: Kinesin superfamily motor proteins and intracellular transport. Nat Rev Mol Cell Biol 10: 682-696, 2009.

10. Miki H, Setou M, Kaneshiro K and Hirokawa N: All kinesin superfamily protein, KIF, genes in mouse and human. Proc Natl Acad Sci USA 98: 7004-7011, 2001.

11. Hirokawa N and Tanaka Y: Kinesin superfamily proteins (KIFs): Various functions and their relevance for important phenomena in life and diseases. Exp Cell Res 334: 16-25, 2015.

12. Lawrence CJ, Dawe RK, Christie KR, Cleveland DW, Dawson SC, Endow SA, Goldstein LS, Goodson HV, Hirokawa N, Howard J, et al: A standardized kinesin nomenclature. J Cell Biol 167: 19-22, 2004

13. Nakagawa T, Tanaka Y, Matsuoka E, Kondo S, Okada Y, Noda Y, Kanai Y and Hirokawa N: Identification and classification of 16 new kinesin superfamily (KIF) proteins in mouse genome. Proc Natl Acad Sci USA 94: 9654-9659, 1997.

14. Bassi ZI, Audusseau M, Riparbelli MG, Callaini G and D'Avino PP: Citron kinase controls a molecular network required for midbody formation in cytokinesis. Proc Natl Acad Sci USA 110: 9782-9787, 2013.

15. Arora K, Talje L, Asenjo AB, Andersen P, Atchia K, Joshi M, Sosa H, Allingham JS and Kwok BH: KIF14 binds tightly to microtubules and adopts a rigor-like conformation. J Mol Biol 426: 2997-3015, 2014.

16. Gruneberg U, Neef R, Li X, Chan EH, Chalamalasetty RB, Nigg EA and Barr FA: KIF14 and citron kinase act together to promote efficient cytokinesis. J Cell Biol 172: 363-372, 2006.

17. Watanabe $S$, De Zan T, Ishizaki T and Narumiya S: Citron kinase mediates transition from constriction to abscission through its coiled-coil domain. J Cell Sci 126: 1773-1784, 2013.

18. Molina I, Baars S, Brill JA, Hales KG, Fuller MT and Ripoll P: A chromatin-associated kinesin-related protein required for normal mitotic chromosome segregation in Drosophila. J Cell Biol 139: 1361-1371, 1997.

19. Carleton M, Mao M, Biery M, Warrener P, Kim S, Buser C, Marshall CG, Fernandes C, Annis J and Linsley PS: RNA interference-mediated silencing of mitotic kinesin KIF14 disrupts cell cycle progression and induces cytokinesis failure. Mol Cell Biol 26: 3853-3863, 2006.

20. Zhu C, Zhao J, Bibikova M, Leverson JD, Bossy-Wetzel E, Fan JB, Abraham RT and Jiang W: Functional analysis of human microtubule-based motor proteins, the kinesins and dyneins, in mitosis/cytokinesis using RNA interference. Mol Biol Cell 16: 3187-3199, 2005. 
21. Moawia A, Shaheen R, Rasool S, Waseem SS, Ewida N, Budde B Kawalia A, Motameny S, Khan K, Fatima A, et al: Mutations of KIF14 cause primary microcephaly by impairing cytokinesis. Ann Neurol 82: 562-577, 2017.

22. Makrythanasis P, Maroofian R, Stray-Pedersen A, Musaev D, Zaki MS, Mahmoud IG, Selim L, Elbadawy A, Jhangiani SN, Coban Akdemir ZH, et al: Biallelic variants in KIF14 cause intellectual disability with microcephaly. Eur J Hum Genet 26 : 330-339, 2018

23. Huang W, Wang J, Zhang D, Chen W, Hou L, Wu X and Lu Y: Inhibition of KIF14 suppresses tumor cell growth and promotes apoptosis in human glioblastoma. Cell Physiol Biochem 37: $1659-1670,2015$

24. Corson TW, Zhu CQ, Lau SK, Shepherd FA, Tsao MS and Gallie BL: KIF14 messenger RNA expression is independently prognostic for outcome in lung cancer. Clin Cancer Res 13 3229-3234, 2007.

25. Xu H, Choe C, Shin SH, Park SW, Kim HS, Jung SH, Yim SH, Kim TM and Chung YJ: Silencing of KIF14 interferes with cell cycle progression and cytokinesis by blocking the p27(Kip1) ubiquitination pathway in hepatocellular carcinoma. Exp Mol Med 46: e97, 2014.

26. Abiatari I, DeOliveira T, Kerkadze V, Schwager C, Esposito I, Giese NA, Huber P, Bergman F, Abdollahi A, Friess H, et al: Consensus transcriptome signature of perineural invasion in pancreatic carcinoma. Mol Cancer Ther 8: 1494-1504, 2009.

27. Singel SM, Cornelius C, Zaganjor E, Batten K, Sarode VR, Buckley DL, Peng Y, John GB, Li HC, Sadeghi N, et al: KIF14 promotes AKT phosphorylation and contributes to chemoresistance in triple-negative breast cancer. Neoplasia 16: 247-256, 256 e242, 2014. https://doi.org/10.1016/j.neo.2014.03.008.

28. Corson TW and Gallie BL: KIF14 mRNA expression is a predictor of grade and outcome in breast cancer. Int J Cancer 119: 1088-1094, 2006.

29. Singel SM, Cornelius C, Batten K, Fasciani G, Wright WE, Lum L and Shay JW: A targeted RNAi screen of the breast cancer genome identifies KIF14 and TLN1 as genes that modulate docetaxel chemosensitivity in triple-negative breast cancer. Clin Cancer Res 19: 2061-2070, 2013.

30. Thériault BL, Cybulska P, Shaw PA, Gallie BL and Bernardini MQ: The role of KIF14 in patient-derived primary cultures of high-grade serous ovarian cancer cells. J Ovarian Res 7: 123, 2014.

31. Thériault BL, Pajovic S, Bernardini MQ, Shaw PA and Gallie BL: Kinesin family member 14: An independent prognostic marker and potential therapeutic target for ovarian cancer. Int J Cancer 130: 1844-1854, 2012

32. Corson TW, Huang A, Tsao MS and Gallie BL: KIF14 is a candidate oncogene in the 1q minimal region of genomic gain in multiple cancers. Oncogene 24: 4741-4753, 2005.

33. Kumar S, Nag A and Mandal CC: A Comprehensive Review on miR-200c, a promising cancer biomarker with therapeutic potential. Curr Drug Targets 16: 1381-1403, 2015.

34. Mutlu M, Raza U, Saatci Ö, Eyüpoğlu E, Yurdusev E and Sahin Ö: miR-200c: A versatile watchdog in cancer progression, EMT, and drug resistance. J Mol Med (Berl) 94: 629-644, 2016.

35. Livak KJ and Schmittgen TD: Analysis of relative gene expression data using real-time quantitative PCR and the 2(-Delta Delta C(T)) Method. Methods 25: 402-408, 2001.

36. Shin G, Kang TW, Yang S, Baek SJ, Jeong YS and Kim SY: GENT: Gene expression database of normal and tumor tissues. Cancer Inform 10: 149-157, 2011.

37. Rhodes DR, Yu J, Shanker K, Deshpande N, Varambally R, Ghosh D, Barrette T, Pandey A and Chinnaiyan AM: ONCOMINE: A cancer microarray database and integrated data-mining platform. Neoplasia 6: 1-6, 2004.

38. Subramanian A, Tamayo P, Mootha VK, Mukherjee S, Ebert BL, Gillette MA, Paulovich A, Pomeroy SL, Golub TR, Lander ES, et al: Gene set enrichment analysis: A knowledge-based approach for interpreting genome-wide expression profiles. Proc Natl Acad Sci USA 102: 15545-15550, 2005.
39. Mootha VK, Lindgren CM, Eriksson KF, Subramanian A Sihag S, Lehar J, Puigserver P, Carlsson E, Ridderstråle M, Laurila E, et al: PGC-1alpha-responsive genes involved in oxidative phosphorylation are coordinately downregulated in human diabetes. Nat Genet 34: 267-273, 2003.

40. Cerami E, Gao J, Dogrusoz U, Gross BE, Sumer SO, Aksoy BA, Jacobsen A, Byrne CJ, Heuer ML, Larsson E, et al: The cBio cancer genomics portal: An open platform for exploring multidimensional cancer genomics data. Cancer Discov 2: 401-404, 2012.

41. Mermel CH, Schumacher SE, Hill B, Meyerson ML, Beroukhim R and Getz G: GISTIC2.0 facilitates sensitive and confident localization of the targets of focal somatic copy-number alteration in human cancers. Genome Biol 12: R41, 2011.

42. Betel D, Wilson M, Gabow A, Marks DS and Sander C: The microRNA.org resource: Targets and expression. Nucleic Acids Res 36 (Database): D149-D153, 2008.

43. Lewis BP, Burge CB and Bartel DP: Conserved seed pairing, often flanked by adenosines, indicates that thousands of human genes are microRNA targets. Cell 120: 15-20, 2005.

44. Li LT, Jiang G, Chen Q and Zheng JN: Ki67 is a promising molecular target in the diagnosis of cancer (Review). Mol Med Rep 11: 1566-1572, 2015.

45. Liu P, Begley M, Michowski W, Inuzuka H, Ginzberg M, Gao D, Tsou P, Gan W, Papa A, Kim BM, et al: Cell-cycle-regulated activation of Akt kinase by phosphorylation at its carboxyl terminus. Nature 508: 541-545, 2014.

46. Liang J and Slingerland JM: Multiple roles of the PI3K/PKB (Akt) pathway in cell cycle progression. Cell Cycle 2: 339-345, 2003.

47. Thériault BL, Basavarajappa HD, Lim H, Pajovic S, Gallie BL and Corson TW: Transcriptional and epigenetic regulation of KIF14 overexpression in ovarian cancer. PLoS One 9: e91540, 2014.

48. O'Brien SJ, Carter JV, Burton JF, Oxford BG, Schmidt MN, Hallion JC and Galandiuk S: The role of the miR-200 family in epithelial-mesenchymal transition in colorectal cancer: A systematic review. Int J Cancer 142: 2501-2511, 2018.

49. Tian Y, Pan Q, Shang Y, Zhu R, Ye J, Liu Y, Zhong X, Li S, He Y, Chen L, et al: MicroRNA-200 (miR-200) cluster regulation by achaete scute-like 2 (Ascl2): Impact on the epithelialmesenchymal transition in colon cancer cells. J Biol Chem 289: 36101-36115, 2014

50. Lu YX, Yuan L, Xue XL, Zhou M, Liu Y, Zhang C, Li JP, Zheng L, Hong M and Li XN: Regulation of colorectal carcinoma stemness, growth, and metastasis by an miR-200c-Sox2-negative feedback loop mechanism. Clin Cancer Res 20: 2631-2642, 2014.

51. Toiyama Y, Hur K, Tanaka K, Inoue Y, Kusunoki M, Boland CR and Goel A: Serum miR-200c is a novel prognostic and metastasis-predictive biomarker in patients with colorectal cancer. Ann Surg 259: 735-743, 2014.

52. Wang M, Zhang P, Li Y, Liu G, Zhou B, Zhan L, Zhou Z and Sun X: The quantitative analysis by stem-loop real-time PCR revealed the microRNA-34a, microRNA-155 and microRNA$200 \mathrm{c}$ overexpression in human colorectal cancer. Med Oncol 29: 3113-3118, 2012.

53. Hur K, Toiyama Y, Takahashi M, Balaguer F, Nagasaka T, Koike J, Hemmi H, Koi M, Boland CR and Goel A: MicroRNA-200c modulates epithelial-to-mesenchymal transition (EMT) in human colorectal cancer metastasis. Gut 62: 1315-1326, 2013

54. Pan Q, Meng L, Ye J, Wei X, Shang Y, Tian Y, He Y, Peng Z, Chen L, Chen W, et al: Transcriptional repression of miR-200 family members by Nanog in colon cancer cells induces epithelial-mesenchymal transition (EMT). Cancer Lett 392: 26-38, 2017.

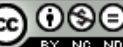

This work is licensed under a Creative Commons Attribution-NonCommercial-NoDerivatives 4.0 International (CC BY-NC-ND 4.0) License. 